OPEN ACCESS

Edited by:

Eckhard Wolf,

Ludwig Maximilian University of

Munich, Germany

Reviewed by:

Dipankar Gupta,

University of Florida, United States

Emanuele Cozzi,

University Hospital of Padua, Italy

*Correspondence:

Muhammad M. Mohiuddin

mmohiuddin@som.umaryland.edu

Specialty section:

This article was submitted to

General Cardiovascular Medicine,

a section of the journal

Frontiers in Cardiovascular Medicine

Received: 21 February 2019

Accepted: 25 June 2019

Published: 25 July 2019

Citation:

DiChiacchio L, Singh AK, Chan JL, Shockcor NM, Zhang T, Lewis BG,

Ayares D, Corcoran P, Horvath KA and Mohiuddin MM (2019) Intra-Abdominal Heterotopic Cardiac

Xenotransplantation: Pearls and

Pitfalls. Front. Cardiovasc. Med. 6:95.

doi: 10.3389/fcvm.2019.00095

\section{Intra-Abdominal Heterotopic Cardiac Xenotransplantation: Pearls and Pitfalls}

\author{
Laura DiChiacchio ${ }^{1}$, Avneesh K. Singh ${ }^{1}$, Joshua L. Chan ${ }^{2}$, Nicole M. Shockcor ${ }^{1}$, \\ Tianshu Zhang ${ }^{1}$, Billeta G. Lewis ${ }^{1}$, David Ayares ${ }^{3}$, Philip Corcoran ${ }^{2}$, Keith A. Horvath ${ }^{2}$ and \\ Muhammad M. Mohiuddin ${ }^{1 *}$
}

${ }^{1}$ Department of Surgery, University of Maryland Medical Center, Baltimore, MD, United States, ${ }^{2}$ National Heart, Lung, Blood Institute, National Institute of Health, Bethesda, MD, United States, ${ }^{3}$ Revivicor, Inc., Blacksburg, VA, United States

Heterotopic cardiac xenotransplantation in the intra-abdominal position has been studied extensively in a pig-to-baboon model to define the optimal donor genetics and immunosuppressive regimen to prevent xenograft rejection. Extensive investigation using this model is a necessary stepping stone toward the development of a life-supporting animal model, with the ultimate goal of demonstrating suitability for clinical cardiac xenotransplantation trials. Aspects of surgical technique, pre- and post-operative care, graft monitoring, and minimization of infectious risk have all required refinement and optimization of heterotopic cardiac xenotransplantation over time. This review details non-immunologic obstacles relevant to this model described by our group and in the literature, as well as strategies that have been developed to address these specific challenges.

Keywords: xenotransplant, xenograft, cardiac, pig, baboon, abdominal, heterotopic, complications

\section{INTRODUCTION}

Heterotopic cardiac xenotransplantation model is widely used to study the immunology of xenograft rejection and to test various immunosuppressive regimens. This model has several advantages as it facilitates immunologic monitoring through the period of graft rejection at reduced cost and complexity compared to orthotopic models. Since the native heart remains in place, rejection of heterotopic xenograft does not result in primary hemodynamic compromise. This model has additionally allowed for the successful demonstration of multiple immunosuppression regimens that have resulted in long-term xenograft survival (1-4). However, due to its non-physiologic nature, it does not offer information as to whether the organ can sustain life.

Multiple challenges affect the successful transplantation of a heterotopic cardiac xenograft. Immunologic complications have been a major hurdle to long-term graft and recipient survival, as both bacterial and fungal infections due to non-specific immune suppression can be commonly seen (5). Viral pathogens, such as cytomegalovirus (CMV) herpes simplex virus (HSV) and simian T-cell leukemia virus (STLV) are common in baboons, although this has been lessened with the implementation of specific-pathogen-free baboons, early weaning, and serologic screening $(6,7)$. All donor pigs are weaned early and demonstrate negative serology for porcine CMV prior to transplant. Thromboembolic complications are also linked to this model, due to de novo cross-species incompatibilities as well as the use of therapeutic immunosuppressive agents, such as anti-CD154 ligand antibody (8-11). Systemic coagulopathies similar to disseminated intravascular 
coagulation (DIC) are also shown to be associated with delayed rejection of pig xenograft in the pig-to-baboon species combination (12). Understanding these common complications as well as the tools with which to overcome them is essential to the success of this model.

This paper describes our group's experience and the lessons learned with the pig-to-baboon intra-abdominal heterotopic cardiac xenotransplant model, with a cohort of 88 transplants to date, and provides a systemic review of available literature. Results from our group are from both the National Institutes of Health and University of Maryland Medical Center and the experiments detailed in this review were approved by the respective Institutional Animal Care and Use Committees.

\section{TECHNICAL CONSIDERATIONS}

The intra-abdominal heterotopic model is a two-anastomosis system utilizing arterial supply from the infrarenal aorta of the recipient baboon to perfuse the coronaries of the donor pig heart with drainage of the xenograft through the donor pulmonary artery remnant anastomosed to the abdominal inferior vena cava of the recipient. During procurement of the donor heart, arrhythmias and prolonged warm ischemia time must be avoided. Pretreatment with amiodarone $(15 \mathrm{mg})$ is our standard for dysrhythmia prophylaxis. Heparinization is achieved with 500 IU/kg $3 \mathrm{~min}$ prior to cross-clamp. Diastolic arrest is achieved with $20-30 \mathrm{cc} / \mathrm{kg}$ of cold crystalloid cardioplegia, followed by cold static ischemia. We generally use Custodial HTK, but equivalent commercial preservation solutions, such as Belzer UW are acceptable.

During implantation, care must be taken to protect bowel and bladder during exposure of the abdominal aorta at the level of the iliac bifurcation. Additionally, geometric considerations during implantation of the graft are key, as kinking of either donor aorta or pulmonary artery can lead to inflow or outflow obstruction that may result in stasis, thrombus formation, and graft failure. The pulmonary artery to inferior vena cava anastomosis is particularly susceptible to thrombosis from angulation at the time of abdominal closure. As in an orthotopic position, deairing of the heart and establishment of normal sinus rhythm are integral to long-term graft survival, and electrocardioversion is occasionally required to establish such a rhythm as described in the arrhythmias in donor heart section below. Adams et al. described early experience with avulsion of the aortic suture line immediately upon post-operative movement of the baboon into an upright position. The authors report that particular care with replacement of bowel loops into anatomic position prior to closure prevented any further disruption of the graft secondary to shifting of intra-abdominal contents (13).

\section{POST-OPERATIVE MANAGEMENT}

A number of supportive measures are required to maintain optimal function of a heterotopic xenograft that would not be utilized in an orthotopic model due to its non-load bearing, relatively low-flow state. Continuous intravenous heparin is required to keep the activated clotting time to twice its baseline level to minimize clot formation. This is particularly imperative with longer surviving xenografts as formation of left ventricular thrombi are more frequently observed. In addition, continuous infusion of heparin has been demonstrated to have a mitigating effect on the development of consumptive coagulopathy, and has been utilized in other models, including the kidney xenograft model (14).

Cefazolin is routinely utilized for bacterial prophylaxis for 1 week following placement of a central venous catheter (Bard Medical, Covington, GA). This line is maintained until the xenograft is explanted to allow for delivery of medications over extended periods of time (e.g., continuous heparin, 2-h infusion of MMF, etc.), access for routine blood draws, or administration of therapies during emergent circumstances. The central venous catheter traverses through a jacket and tether system (Lomir Biomedical, Inc.) that protects it from inadvertent damage by the baboon and allows for access by the research team from outside the baboon cage. The long-term tethering system permits relative mobility of the animal while still providing durable and reliable intravenous access for the research team (15). Over time, multiple improvements have been implemented, including improvement in the durability of the jackets, strengthening the critical attachment of tether to the jacket, and redesigning the swivel system to reduce friction. We have additionally identified that providing a period of time for the animal to acclimatize to both jacket and tether prior to the start of the experiment improved the lifespan of the system.

Implanted telemetry (DSI, St. Paul, MN) as described elsewhere (16) is utilized for early detection of infection, in the form of central temperature monitoring, and graft rejection, through measurement of left ventricular pressure and continuous electrocardiography. This has been utilized for monitoring of continuous EKG in this model since its inception (13). Interval monitoring with blood work, manual palpation of the graft, as well as graft echocardiography is undertaken. Close monitoring allows for prompt treatment of developing infection, thrombus formation and in rare cases graft rejection. In cases where telemetry, laboratory, physical exam, and ultrasonography suggest rejection, there is a role for open biopsy in this model. This is done only when absolutely necessary per institutional animal care committee restrictions and is generally performed at the time of potential explant.

\section{WEIGHT MATCHING FOR THE INTRA-ABDOMINAL POSITION}

The disparity between the donor and the recipient size plays an important role in subsequent development of complications. A heart from a smaller pig (3-4 weeks old $\sim 5-6 \mathrm{~kg}$ in weight) transplanted in a larger baboon $(>10 \mathrm{~kg})$ allows enough room for the heart to function without compression and fibrillation at abdominal closure. On the other hand, a larger heart in a smaller baboon appears to compress the graft, restricting its systolic function, abdominal compartment syndrome with hypotension of the recipient and induces arrhythmias leading 
to loss of the xenograft if not recognized and treated promptly. The relatively small size of available recipient baboons and our preference to use smaller baboons to save on costly immunosuppressive drugs limits the size of potential donor pig hearts. In our group's experience, grafts from larger donor pigs become compressed by the recipient's abdominal wall during closure, resulting in elevated abdominal compartment pressures. Figure 1 compares survival of grafts based on donor and recipient weights. The disparity between the donor and the recipient size plays an important role in development of subsequent complications. As a general rule, a donor pig of $\sim 80 \%$ of the recipient baboon weight allows enough room for the heart to function without compression at abdominal closure. Xenografts greater than this size ratio frequently resulted in decreased systolic function, abdominal compartment syndrome, and lethal cardiac arrhythmias.

Size matching in the heterotopic model has always been a challenge, even prompting early groups to utilize cervical implantation as an alternative $(13,17)$. While case reports of successful transplantation with a donor-recipient weight mismatch of more than $20 \%$ have been published, other series demonstrate that a donor:recipient weight ratio of $<0.66$ results in increasing morbidity and mortality in the recipient (18). Another consideration is the health and intrinsic cardiac function of transgenic pigs at various sizes; this is a potential confounder of these outcomes. Nevertheless, we have found that with further experience in size matching, and technology for non-invasive graft monitoring, abdominal heterotopic placement of the cardiac xenograft has become easily accessible and manageable.

\section{BLEEDING COMPLICATIONS}

Hemorrhagic complications in our experience are attributed to several causes. Consumptive coagulopathy is a known sequela of xenograft placement and generally occurs within 4 weeks of transplantation. In our early experience, this occurred in $\sim 10 \%$ of our cases and is discussed in more detail in Coagulation Dysfunction, below. The need for continuous

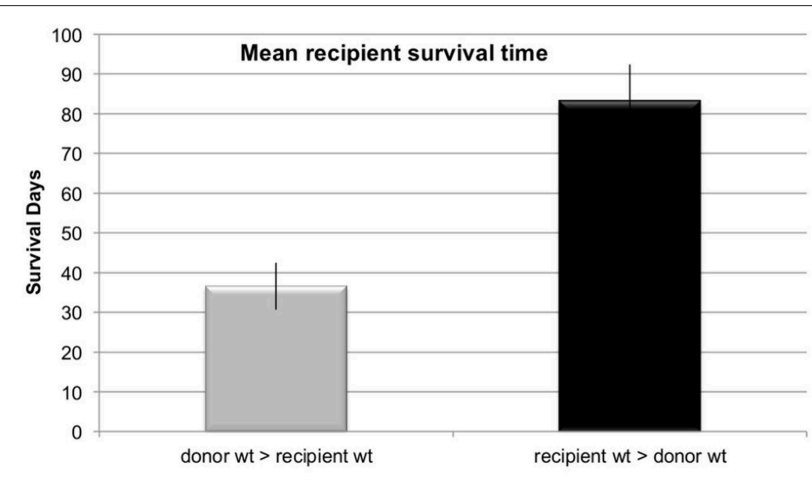

FIGURE 1 | Weight matching donors and recipients. Mean recipient survival, in days, stratified by donor weight greater than or less than recipient weight, respectively. heparin infusion further increases the risk of bleeding in the xenotransplant setting.

In some initial experiments, the site of the LV telemetry probe bled; this was prevented in later transplants by securing the probe tightly with pledgetted Prolene sutures (Figure 2A). In two cases a region of the left ventricle failed to reperfuse, became infarcted, and ruptured within 2-3 weeks, leading to recipient fatality (Figure 2B). In one case bleeding was the result of a ruptured mesenteric vessel that had adhered to the transplanted graft. There was no technical error identified in this case on necropsy. In our earlier cases when anti-inflammatory drugs were not used, mesenteric and bowel adhesions around the transplanted heart were a common occurrence as described below, and in one very active baboon it led to bleeding from these adhesions.

\section{THROMBOEMBOLIC COMPLICATIONS}

Use of costimulation blockade of the CD40/CD154 pathway for induction and maintenance immunosuppressive therapy has led to the recent profound prolongation of graft survival. Early experience utilizing anti-CD154 antibody was successful in immunomodulation but led to an unacceptable incidence of complications from thromboembolic events (11). Phase II trials using anti-CD154 antibody to treat lupus and kidney transplant recipients were forced to end early due to adverse events from thromboembolic complications (19, 20). These effects are thought to be secondary to antibody-binding of CD154 expressed on activated platelets, increasing platelet aggregation and thrombus formation $(10,11)$. Early use of anti-CD154 in our group utilized ketorolac in conjunction to decrease the degree of platelet aggregation has had encouraging results. We have now transitioned to the use of anti-CD40 antibody in place of antiCD154 for costimulation blockade. This has significantly reduced the rate of thromboembolic complications while maintaining

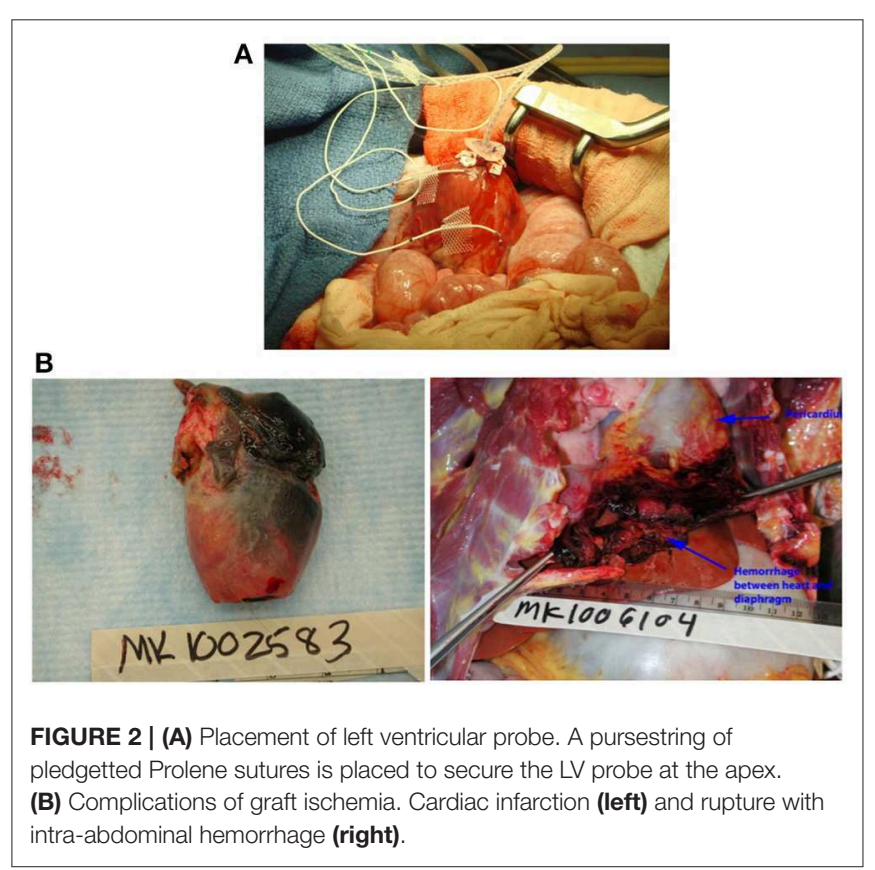


effective immunosuppression and favorable xenograft survival (21). In addition, anticoagulation with systemic unfractionated heparin is used routinely in our recipients to target an activated clotting time $2 \times$ the baseline to prevent thrombus formation.

\section{INTESTINAL ADHESION AND RELATED COMPLICATIONS}

In addition to the bleeding associated with the single case of adhesed mesenteric vessel rupture, intestinal adhesions are a risk specific to this model. While adhesions are a risk associated with any abdominal operation, the inflammation associated with xenograft placement confers a particularly high risk of significant adhesion formation. As shown in Figure 3, adhesions of omentum and small bowel to the xenograft were observed in almost all transplants; in a few cases, these adhesions were sufficiently severe to contribute to recipient fatality. One intestinal obstruction led to aspiration and sudden death; another very active, otherwise healthy recipient expired as a result of intestinal perforation. Theoretically, an adhesion barrier could be utilized to minimize the risks associated with intra-abdominal adhesion formation; personal experience with adhesion barriers have shown no difference in adhesion formation, likely due to the presence of exceptionally high levels of inflammation in this model. We have seen markedly reduced adhesions lately as we have routinely started using anti-inflammatory drugs in our regimen, including IL-6R and $\mathrm{TNF} \alpha$ inhibitors.

\section{ARRHYTHMIAS IN THE DONOR HEART}

Atrial and ventricular fibrillation, ventricular tachycardia and bundle branch block were witnessed in conditions when larger hearts were used in relatively small baboons. Pig hearts, and genetically-modified (GM) pig hearts in particular, are more prone to fibrillation than human or non-human primate hearts. Subtle differences in ion channel expression in the GM pig heart are hypothesized to account for this increased myocardial

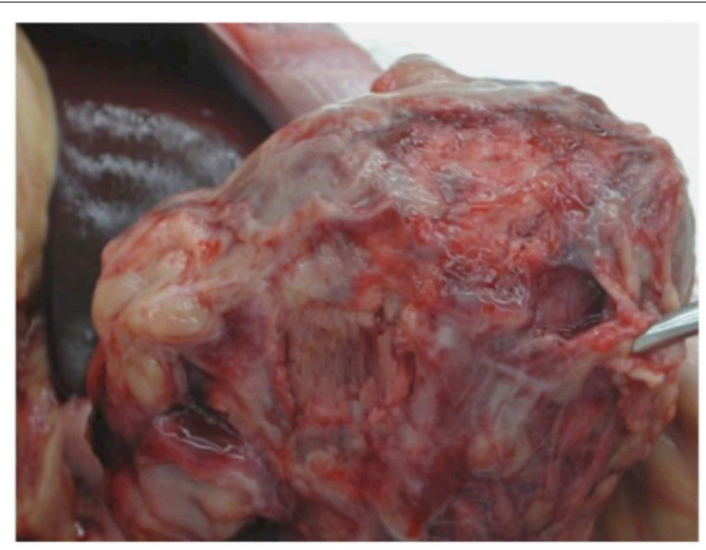

FIGURE 3 | Formation of adhesions. Dense, diffuse intestinal adhesions apparent surrounding graft. irritability (22). We utilize a cocktail of mannitol, lidocaine, and sodium bicarbonate during reperfusion to reduce this risk. When fibrillation does present, it occurs immediately after reperfusion, during the closure of the abdominal cavity, or within $12 \mathrm{~h}$ post-operatively. Arrhythmias are treated with an amiodarone bolus ( $15 \mathrm{mg}$ ) followed by initiation of a continuous amiodarone infusion $(15 \mathrm{mg} / 24 \mathrm{~h})$ and electrical cardioversion as necessary. Because electrocardioversion results in the longterm telemetry device becoming inoperable due to the spike in current, cardioversion was not used in a number of late cases of fibrillation, resulting in loss of graft function without rejection in all of these cases. In some cases, when detected early, the implant was removed, the heart cardioverted into sinus rhythm, the device re-implanted and the abdomen closed without further incident.

\section{COAGULATION DYSFUNCTION}

Two types of disorders were observed related to dysregulated coagulation. The consumptive coagulopathy typically seen in this model has been described by others (23-25); however, platelet consumption and uncontrollable bleeding were only observed in two baboons with functioning grafts. One baboon demonstrated hypercoagulability without thrombocytopenia or hemorrhage, which manifested as thrombosis in jugular vessels repeatedly leading to occlusion of two IV catheters. A very large dose of heparin infusion $(1,000 \mathrm{U} / \mathrm{h}$ compared to $50 \mathrm{U} / \mathrm{h}$ usual dose for an $8 \mathrm{~kg}$ baboon) was required to prevent coagulation and recurrent line thrombosis. Two baboons experienced a sharp decrease in platelet count and hematocrit 21 and 28 days after transplant, respectively, and responded well to transfusion of allogeneic packed red blood cells. However, more than three transfusions also precipitated severe coagulopathy leading to recipient mortality. A tight control of ACT and platelet count in two baboons is demonstrated in Figure 4.

We observed a drop in hematocrit (HCT) to $15 \%$ in four recipients who also developed bruising over their abdomen. The platelet numbers in these baboons also dropped to $50-70,000 / \mathrm{mm}^{3}$. Initially small amounts of packed red cell transfusion $(60-70 \mathrm{ml})$ transiently improved the condition but was not able to sustain the affect. Later we were able to transfuse large quantities of blood ( $20 \mathrm{ml} / \mathrm{kg}$ packed $\mathrm{RBC}$ 's) that greatly helped in restoring the HCT to normal. Over a period of time, platelet numbers also improved in these baboons. In three cases where multiple ( $>2 ; 8-20 \mathrm{ml}$ packed $\mathrm{RBC} / \mathrm{kg}$ ) blood transfusions were deemed clinically necessary the recipient succumbed in association with uncontrolled consumptive coagulopathy.

\section{INFECTIOUS COMPLICATIONS}

Infectious complications are of paramount significance in the immunosuppressed recipient. Routine use of prophylactics (Cefazolin for the first week following transplant and ganciclovir throughout the duration of immunosuppression) is mandatory in our model. Additionally, long-term telemetry device placement allows for continuous body temperature recording 


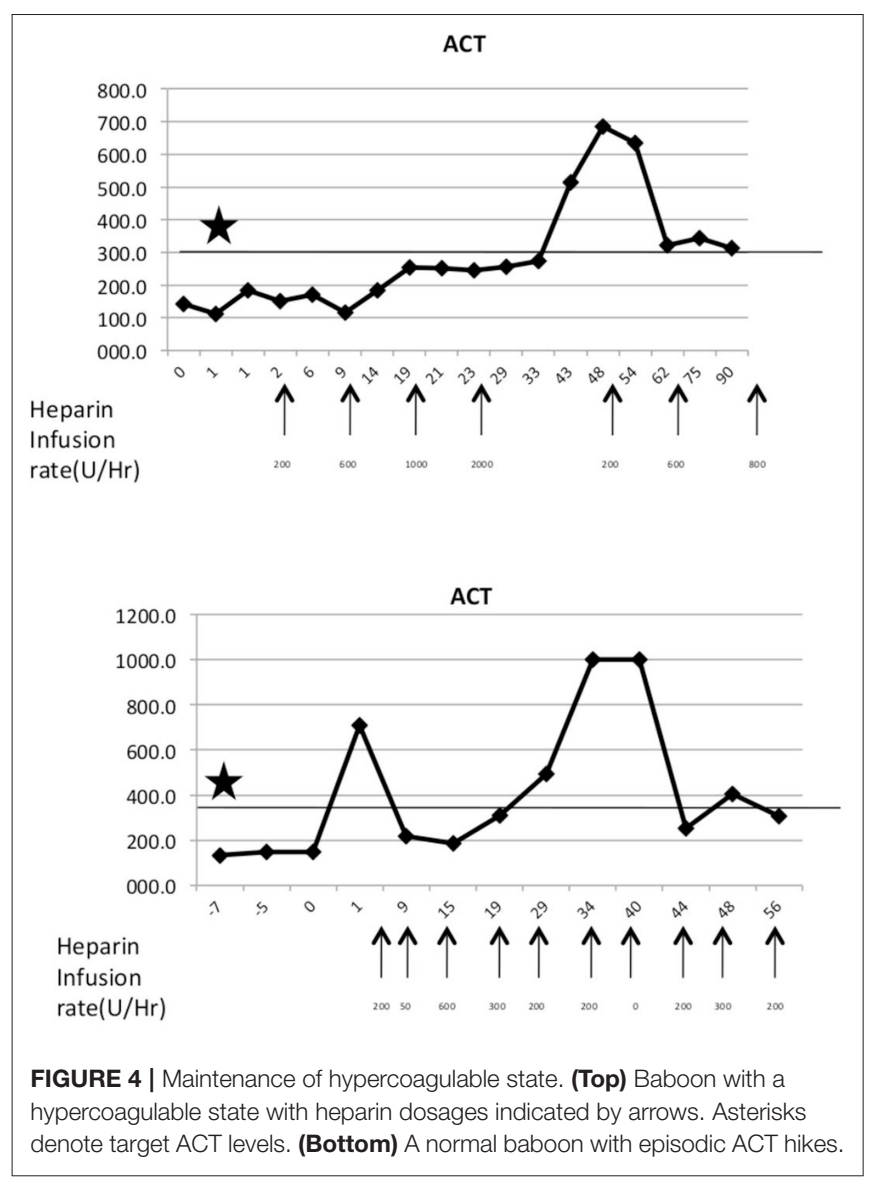

such that any febrile episodes followed by blood culture were promptly evaluated and treated. In fact, the benefit of the implanted telemetry device, primarily designed for direct pressure monitoring of the graft, was primarily through the early detection of central temperature spikes. This allowed us to overcome the majority of potential infections complications efficiently with the use of broad-spectrum antibiotics in any recipient exhibiting a fever $>39^{\circ} \mathrm{C}$. One case of fungal infection (Zygomycosis) did occur that was ultimately diagnosed by identification of fungus on histological analysis (Figure 5). No clinically significant Cytomegalovirus infections occurred. While attention to the long-term tunneled central line is important, we do not routinely exchange these lines, nor have we experienced line-related infections in this model.

One early series, prior to the use of implantable telemetry and continuous temperature monitoring, demonstrated 23 episodes of infection in a series of 16 baboons; 14 of these episodes resolved with treatment, although unfortunately 8 resulted in death of the recipient (5). In this series the mean time from transplant to infection was 44 days. All CMV infections (6) were with baboon $\mathrm{CMV}$ and were associated with low levels of ganciclovir. No episodes of fungal infection were observed in this series.

We have found with the use of early intervention based on results from continuous non-invasive monitoring that clinically significant infections are rare, and controlled effectively with bacterial, viral, and fungal prophylaxis, as well as prompt treatment of fever with broad-spectrum coverage $(26,27)$.

\section{SUDDEN DEATH}

Two baboons suffered sudden death without any prior sign and symptoms. Necropsy excluded acute hemorrhage, pulmonary embolus, pneumonia, and intra-abdominal catastrophe, but did not yield any conclusive evidence regarding the cause of death. Ventricular fibrillation is a potential etiology that could not be excluded on post-mortem exam.

\section{CONCLUSIONS}

The heterotopic transplant model has served as a useful tool to study the immunological barriers to xenotransplantation. This model has also allowed testing and refinement of several immunosuppressive and immunomodulatory agents and approaches, and demonstrated the clear benefits associated with various genetic modifications to donor pigs. While a number of complications remain in this model, the immunologic success has formed the foundation for experiments in the orthotopic model. The successful survival of the intra-abdominal heterotopic cardiac xenograft requires vigilance by the scientific team; so, too, will success in the orthotopic model.

We recently reported the longest survival of 945 days in a heterotopic xenotransplantation model utilizing either GTKO pigs that also express human CD46 and thrombomodulin (GTKO.hCD46.TBM) $(21,28)$. That result was achieved using an immunosuppressive regimen that is a combination of regimens reported earlier, based on co-stimulation blockade with antiCD40, MMF and anti-CD20 antibody. Herein, we describe challenges in the use of this model that are unrelated to immunosuppression. We have little direct evidence that the immunosuppression, which appeared to have been largely sufficient to surpass the anti-non-Gal and other immune barriers, was lethal to the recipient, since viral and bacterial infections were not common. Recipient morbidity and mortality were in many cases attributable to the heterotopic placement of the heart and possibly to the inflammatory rejection process. Initially, a number of recipients in this experiment died before complete rejection of the heart xenograft due to some avoidable complications. Important lessons were learned from these experiments and these complications were prevented from recurring.

Some of the early hurdles were related to the tether, animal jacketing and jugular intravenous catheters. These were overcome following several modifications. We faced the problem of consumptive coagulopathy and thromboembolism in a few baboons but mostly these complications occurred within the first post-transplantation month. Some of the late complications included sudden death, uncommon infections and abdominal bleeding due to rupture of the transplanted heart or mesenteric vessels adherent to the xenograft. While the animals did undergo multiple procedures with multiple cardiac stressors, no evidence 
i

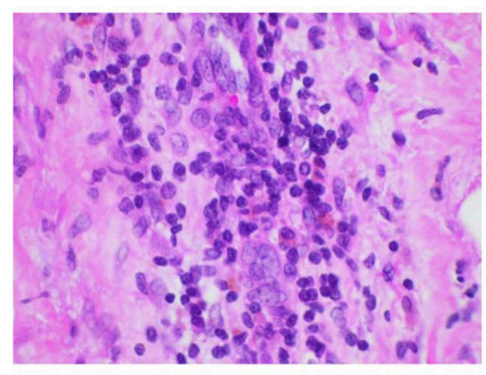

ii.

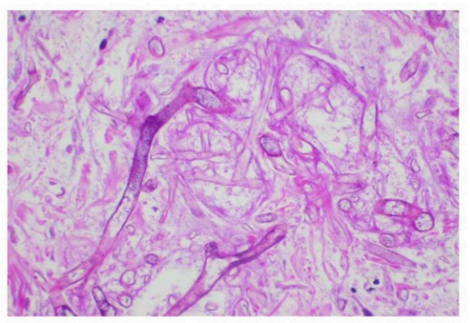

iii.

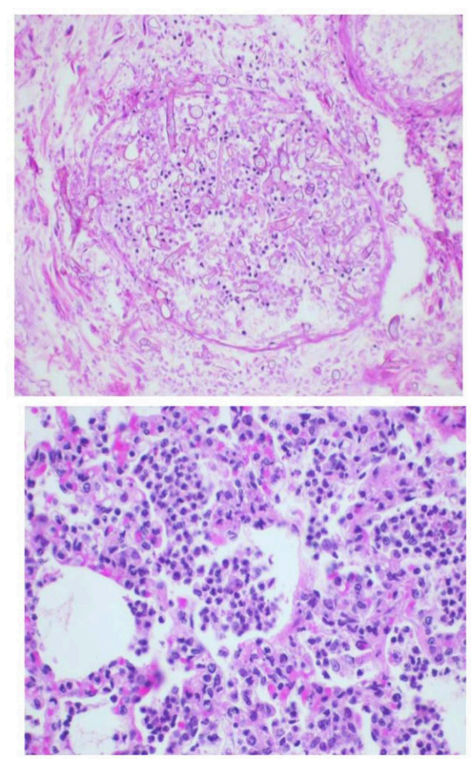

FIGURE 5 | Histologic evidence of infections. (i) Lymphocytic infiltrates in transplanted heart; (ii, iii) Zygomycosis; and (iv) bronchopneumonia.

of native cardiomyopathy or depressed myocardial function in the native heart was observed in our experiments.

Typical consumptive coagulopathy reported by many was not common in our experiment. We did notice signs similar to consumptive coagulopathy in two baboons. These symptoms were overcome in one case with a series of packed red cell infusions to restore normal coagulation profile. However, three other cases resulted in demise of these baboons. Based on this, we recommend that persistent or recurrent anemia in this model should be treated by graft removal or euthanasia. The coagulopathy observed was limited to a sharp decrease in platelet numbers and elevation of activated clotting time of blood. This early drop in platelet numbers is likely due to deposition in the graft. When the graft survives this insult, the numbers normalize on their own. This is inconsistent with an immunemediated process, such as heparin-induced antibody mediated thrombocytopenia, which we have not seen in our experiments.

In one case thrombosis of the intravenous catheter and subsequent occlusion of a major vessel also hampered the success of this experiment. Some baboons required higher doses of heparin infusion (up to 2,000 U/h) to achieve target ACT levels of $2 \times$ baseline. Thrombotic microangiopathy related to anti CD154 has been described (29) but in our study we did not come across this complication in the absence of graft dysfunction. Perhaps routine administration of ketorolac immediately before the administration of this antibody prevented this complication as previously reported (7).

Intestinal adhesions to the heterotopic heart caused recipient mortality in two recipients, due to bowel obstruction with aspiration, intestinal rupture, and mesenteric vessel erosion. These complications led to recipient baboon's mortality before the transplanted heart was rejected. In additional cases, bowel obstruction due to intestinal adhesions was relieved surgically when identified early by abdominal X-ray, and we recommend use of X-ray imaging early for any baboon with difficulty tolerating a diet, lethargy, or signs of discomfort or systemic illness without decreased graft function.

The use of telemetry implant helped accurate measurement of graft function and facilitated early detection of graft dysfunction (ventricular fibrillation, decreased contractility in association with immunologic injury). The heart rate and ventricular contractility based on left ventricular peak systolic and end diastolic pressure accurately and non-invasively measured graft function. The temperature probe was very useful in detecting the early onset of infection and these infections were treated at an early stage. Therefore, this complication was not a significant factor in our experiments, in contrast to experiences reported by others (5). Continuous treatment with intravenous Ganciclovir also was effective to prevent baboon CMV infection.

In conclusion, long-term xenograft survival has been achieved in a large animal heterotopic model where the loss of recipients to model-associated complications often used to occurs before the loss of graft function. While most of these complications are avoided in our recent experiments, we conclude that, based on encouraging results with current GM pig lines and immunosuppressive regimens, the orthotopic model may offer new challenges but a more productive format as the basis for developing a clinically applicable regimen. With continued vigilance and experience with the intra-abdominal heterotopic model, with new GM pigs and novel target specific immunosuppressive agents, even further advancement toward a successful orthotopic xenotransplant model is within reach. 


\section{DATA AVAILABILITY}

All datasets generated for this study are included in the manuscript and/or the supplementary files.

\section{ETHICS STATEMENT}

Results from our group are from both the National Institutes of Health and University of Maryland Medical Center and the experiments detailed in this review were approved by the respective Institutional Animal Care and Use Committees.

\section{AUTHOR'S NOTE}

MM, AS, BL, and LD are part of the Cardiac Xenotransplantation Program at the University of Maryland.

\section{REFERENCES}

1. Hisashi Y, Yamada K, Kuwaki K, Tseng YL, Dor FJ, Houser SL, et al. Rejection of cardiac xenografts transplanted from alpha1,3-galactosyltransferase geneknockout (GalT-KO) pigs to baboons. Am J Transplant. (2008) 8:2516-26. doi: 10.1111/j.1600-6143.2008.02444.x

2. McGregor CG, Davies WR, Oi K, Teotia SS, Schirmer JM, Risdahl JM, et al. Cardiac xenotransplantation: recent preclinical progress with 3month median survival. J Thorac Cardiovasc Surg. (2005) 130:844-51. doi: 10.1016/j.jtcvs.2005.04.017

3. Tseng YL, Kuwaki K, Dor FJ, Shimizu A, Houser S, Hisashi Y, et al. alpha1,3Galactosyltransferase gene-knockout pig heart transplantation in baboons with survival approaching 6 months. Transplantation. (2005) 80:1493-500. doi: 10.1097/01.tp.0000181397.41143.fa

4. Mohiuddin MM, Corcoran PC, Singh AK, Azimzadeh A, Hoyt RF, Thomas ML, et al. B-cell depletion extends the survival of GTKO.hCD46Tg pig heart xenografts in baboons for up to 8 months. Am J Transplant. (2012) 12:763-71. doi: 10.1111/j.1600-6143.2011.03846.x

5. Teotia SS, Walker RC, Schirmer JM, Tazelaar HD, Michaels MG, Risdahl JM, et al. Prevention, detection, and management of early bacterial and fungal infections in a preclinical cardiac xenotransplantation model that achieves prolonged survival. Xenotransplantation. (2005) 12:127-33. doi: 10.1111/j.1399-3089.2005. 00205.x

6. Wolf RF, Eberle R, White GL. Generation of a specific-pathogen-free baboon colony. J Am Assoc Lab Anim Sci. (2010) 49:814-20.

7. Mueller NJ, Kuwaki K, Dor FJ, Knosalla C, Gollackner B, Wilkinson RA, et al. Reduction of consumptive coagulopathy using porcine cytomegalovirus-free cardiac porcine grafts in pig-to-primate xenotransplantation. Transplantation. (2004) 78:1449-53. doi: 10.1097/01.TP.0000141361.68446.1F

8. Bühler L, Basker M, Alwayn IP, Goepfert C, Kitamura H, Kawai T, et al. Coagulation and thrombotic disorders associated with pig organ and hematopoietic cell transplantation in nonhuman primates. Transplantation. (2000) 70:1323-31. doi: 10.1097/00007890-200011150-00010

9. Kawai T, Andrews D, Colvin RB, Sachs DH, Cosimi AB. Thromboembolic complications after treatment with monoclonal antibody against CD40 ligand. Nat. Med. (2000) 6:114. doi: 10.1038/72162

10. Pinelli DF, Ford ML. Novel insights into anti-CD40/CD154 immunotherapy in transplant tolerance. Immunotherapy. (2015) 7:399-410. doi: 10.2217/imt.15.1

11. Zhang T, Pierson RN, Azimzadeh AM. Update on CD40 and CD154 blockade in transplant models. Immunotherapy. (2015) 7:899-911. doi: 10.2217/IMT.15.54

12. Ierino FL, Kozlowski T, Siegel JB, Shimizu A, Colvin RB, Banerjee PT, et al. Disseminated intravascular coagulation in association with the delayed

\section{AUTHOR CONTRIBUTIONS}

LD participated in the performance of the research and wrote the paper. AS participated in the design and performance of the research and participated in writing and editing the paper. JC participated in the performance of the research, reviewed and edited the paper. NS and TZ participated in the performance of the research and writing the paper. BL participated in the design and performance of the research. DA, PC, and KH participated in the design of the research and in the final approval of the paper. MM designed and performed the research, participated in writing the paper, and provided final approval of the paper.

\section{FUNDING}

MM: NIAID/NIH Grant \#5U19AI090959-10. rejection of pig-to-baboon renal xenografts. Transplantation. (1998) 66:143950. doi: 10.1097/00007890-199812150-00006

13. Adams DH, Chen RH, Kadner A, Naficy S. Technique for heterotopic pig heart xenotransplantation in primates. Ann Thorac Surg. (1999) 68:265-8. doi: 10.1016/S0003-4975(99)00488-9

14. Ezzelarab M, Garcia B, Azimzadeh A, Sun H, Lin CC, Hara H, et al. The innate immune response and activation of coagulation in $\alpha 1,3$-galactosyltransferase gene-knockout xenograft recipients. Transplantation. (2009) 87:805-12. doi: 10.1097/TP.0b013e318199c34f

15. Lukas SE, Griffiths RR, Bradford LD, Brady JV, Daley L. A tethering system for intravenous and intragastric drug administration in the baboon. Pharmacol Biochem Behav. (1982) 17:823-9. doi: 10.1016/0091-3057(82)90366-5

16. Horvath KA, Corcoran PC, Singh AK, Hoyt RF, Carrier C, Thomas $\mathrm{ML}$, et al. Left ventricular pressure measurement by telemetry is an effective means to evaluate transplanted heart function in experimental heterotopic cardiac xenotransplantation. Transplant Proc. (2010) 42:2152-5. doi: 10.1016/j.transproceed.2010.05.117

17. Michler RE, McManus RP, Smith CR, Sadeghi AN, Rose EA. Technique for primate heterotopic cardiac xenotransplantation. J Med Primatol. (1985) 14:357-62.

18. Kadner A, Chen RH, Adams DH. Heterotopic heart transplantation: experimental development and clinical experience. Eur J Cardio Thorac Surg. (2000) 17:474-81. doi: 10.1016/S1010-7940(00)00362-6

19. Boumpas DT, Furie R, Manzi S, Illei GG, Wallace DJ, Balow JE, et al. A short course of BG9588 (anti-CD40 ligand antibody) improves serologic activity and decreases hematuria in patients with proliferative lupus glomerulonephritis. Arthritis Rheum. (2003) 48:719-27. doi: 10.1002/art.10856

20. Vincenti F. New monoclonal antibodies in renal transplantation. Minerva Urol Nefrol. (2003) 55:57-66.

21. Mohiuddin MM, Singh AK, Corcoran PC, Thomas ML III, Clark T, Lewis BG, et al. Chimeric 2C10R4 anti-CD40 antibody therapy is critical for longterm survival of GTKO.hCD46.hTBM pig-to-primate cardiac xenograft. Nat Commun. (2016) 7:11138. doi: 10.1038/ncomms11138

22. Lelovas PP, Kostomitsopoulos NG, Xanthos TT. A comparative anatomic and physiologic overview of the porcine heart. J Am Assoc Lab Anim Sci. (2014) 53:432-8.

23. Robson SC, Candinas D, Siegel JB, Kopp C, Millan M, Hancock WW, et al. Potential mechanism of abnormal thromboregulation in xenograft rejection: loss of ecto-ATPases upon endothelial cell activation. Transplant Proc. (1996) 28:536.

24. Shimizu A, Hisashi Y, Kuwaki K, Tseng YL, Dor FJ, Houser SL, et al. Thrombotic microangiopathy associated with humoral rejection of cardiac xenografts from alpha1,3-galactosyltransferase gene-knockout pigs in baboons. Am J Pathol. (2008) 172:1471-81. doi: 10.2353/ajpath.2008.070672 
25. O'Connell PJ. Thrombotic microangiopathy: the next big hurdle for xenotransplantation. J Am Soc Nephrol. (2005) 16:2529-30. doi: 10.1681/ASN.2005070735

26. Corcoran PC, Horvath KA, Singh AK, Hoyt RF, Thomas ML, Eckhaus MA, et al. Surgical and nonsurgical complications of a pig to baboon heterotopic heart transplantation model. Transplant Proc. (2010) 42:2149-51. doi: 10.1016/j.transproceed.2010.05.116

27. Byrne GW, McGregor CG. Cardiac xenotransplantation: progress and challenges. Curr Opin Organ Transplant. (2012) 17:148-54. doi: 10.1097/MOT.0b013e3283509120

28. Singh AK, Chan JL, DiChiacchio L, Hardy NL, Corcoran PC, Lewis BGT, et al. Cardiac xenografts show reduced survival in the absence of transgenic human thrombomodulin expression in donor pigs. Xenotransplantation. (2018) 5:e12465. doi: 10.1111/xen.12465

29. Buhler L, Alwayn IP, Appel JZ III, Robson SC, Cooper DK. Anti-CD154 monoclonal antibody and thromboembolism. Transplantation. (2001) 71:491. doi: 10.1097/00007890-200102150-00028
Conflict of Interest Statement: DA is the CEO and President of Revivicor, Inc. The authors declare that funding was received from United Therapeutics Inc for this project. The manuscript was also approved for submission by United Therapeutics. The funder had no role in study design, data collection, data analysis, and preparation of manuscript. However, the main material (donor pigs) were provided by them and based on our agreement with them, they had to approve the manuscript for publication. All authors had part of their salary driven from this funding but had no other potential conflict of interest.

Copyright (C) 2019 DiChiacchio, Singh, Chan, Shockcor, Zhang, Lewis, Ayares, Corcoran, Horvath and Mohiuddin. This is an open-access article distributed under the terms of the Creative Commons Attribution License (CC BY). The use, distribution or reproduction in other forums is permitted, provided the original author(s) and the copyright owner(s) are credited and that the original publication in this journal is cited, in accordance with accepted academic practice. No use, distribution or reproduction is permitted which does not comply with these terms. 